\title{
String in Hořava-Lifshitz Gravity
}

\author{
J. Klusoň \\ Department of Theoretical Physics and Astrophysics \\ Faculty of Science, Masaryk University \\ Kotlářská 2, 611 37, Brno \\ Czech Republic \\ E-mail: klu@physics.muni.cz
}

\begin{abstract}
We generalize recent analysis of the dynamics of point particle in HořavaLifshitz background to the case of string probe when we replace the Hamiltonian constraint of the Polyakov string with the constraint that breaks Lorentz invariance of target spacetime. Then we find corresponding Lagrangian and argue that the world-sheet theory is invariant under foliation preserving diffeomorphism. Finally we discuss the Hamiltonian dynamics and show that this is well defined on condition that the world-sheet lapse function obeys the projectability condition.
\end{abstract}

KEYwords: Bosonic String, Hořava-Lifshitz Background. 


\section{Contents}

1. Introduction and Summary 1

2. Dynamics of point particle in Hořava-Lifshitz gravity 2

3. Lorentz Breaking String Theory 5

3.1 Hamiltonian formulation of Polyakov Action

3.2 Lorentz Breaking String Action

3.3 Symmetries of the action 0

4. Hamiltonian Formalism of Non-Relativistic String Theory Revised 12

\section{Introduction and Summary}

Recently Petr Hořava proposed new intriguing approach for the formulation of UV finite quantum theory of gravity [1, 2, 3. 3. ${ }^{1}$. The basic idea of this theory is to modify the UV behavior of the general theory so that the theory is perturbatively renormalizable. However this modification is only possible on condition when we abandon Lorentz symmetry in the high energy regime: in this context, the Lorentz symmetry is regarded as an approximate symmetry observed only at low energy.

It is important to stress that there are two classes of Hořava-Lifshitz theories. One is the class of the projectable theories that are characterized by property that the lapse function is restricted to depend on time only. The other one is the non-projectable class where the lapse is allowed to depend on both space and time. Very nice discussion of the consistency of the non-projectable Hořava-Lifshitz theory was performed in [57] that suggests inconsistency of given theory. For that reason it seems that the projectable version of Hořava-Lifshitz theory is the only consistent one.

The results derived during the analysis of Hořava-Lifshitz gravities imply that it is an interesting problem to study properties of theories with broken general covariance. One such an interesting example of Lorentz breaking theory is the Hamiltonian formulation of the point particle in Hořava-Lifshitz theory that was recently analyzed in [72, 73, 74, 75, 76, 77]. The basic idea of this Lorentz breaking Hamiltonian formalism is that the time and spatial components of momenta are treated differently with very interesting consequences for the notion of causality and formation of black holes.

\footnotetext{
${ }^{1}$ This conjecture was then studied in several papers, see for example [4, 月, 6, 7, 8, 9, 10, 11, 12, 13, 14 , $15,16,17,18,19,20,21,22,23,24,25,26,27,28,29,30,31,32,33,34,35,36,37,38,39,40,41,42,43$, $44,45,46,47,48,76,49,50,51,52,53,54,55,56,57,58,59,60,61,62,64,65,66,63,67,66,69,60,61$.
} 
As the next logical step in this direction is to try to implement this approach for the construction of extended objects, as for example string. The goal of this paper is to proceed in this direction and construct new string theories that we call as Lorentz-breaking string theories (LBS). Explicitly, we study properties of two dimensional gravity coupled to the scalar field when the Hamiltonian constraint breaks the target space Lorentz invariance and analyze its consequence for dynamics of given string. As opposite to the relativistic case when the step from the point particle action to string action is straightforward in case it turns out that in case of LBS there are several ways how to do it. We begin with the Polyakov action and find its Hamiltonian formulation. Then we replace the Hamiltonian constraint with its Lorentz breaking generalization. Since now the world-sheet modes are spatial dependent we find more possibilities how to construct such a Hamiltonian constraint as opposite to the case of the point particle in Hořava-Lifshitz background. The characteristic property of the classical Polyakov action is that the two dimensional gravity is non dynamical. On the other hand we will argue that for the consistency of the LBS theory it is necessary that the spatial component of the world-sheet metric becomes dynamical. Further, we also show that the resulting world-sheet theory is not invariant under full two dimensional diffeomorphism but only under the world-sheet foliation preserving one. Finally, the consistency of the Hamiltonian dynamics of LBS theory again implies that the world-sheet lapse has to obey the projectability condition which is similar result as in case of Hořava-Lifshitz gravity.

Let us conclude our results. We construct new form of string theories with broken target space Lorentz invariance. We should however stress that these string theories have to be considered as toy models for the study of the systems with breaking symmetries. For example, since LBS theories are manifestly non-linear even in flat space-time it is not clear how to formulate their quantum version. In fact, since it is not known whether HořavaLifshitz gravity can be formulated in the framework of string theory it is also questionable how to formulate the action for string probe in given background. However despite of these doubts we believe that the study of such system is interesting in its own and should be extended in may ways. In particular, we would like to see how these LBS theories behave under target space T-duality transformations. We would like also perform similar analysis in case of higher dimensional extended systems, as Dp-branes. We hope to return to these problems in future.

The organization of this paper is as follows. In the next section (2) we review the Hamiltonian and Lagrangian formulation of the probe particle in Horava-Lifshitz background. Then in section (3) we study the string probe in given background and formulate LBS theories. In section (4) we study their Hamiltonian formalism and calculate the algebra of constraints.

\section{Dynamics of point particle in Hořava-Lifshitz gravity}

In this section we review the Hamiltonian dynamics of the particle in Horava-Lifshitz gravity. This section can be considered as the review and extension of analysis performed in $72,73,74,75,76,77$. 
Let us consider space-time $\mathcal{M}$ labeled with coordinates $t, \mathbf{x}=\left(x^{1}, \ldots, x^{D}\right)$ with the metric in $\mathrm{ADM}$ form

$$
g_{00}=-N^{2}+N_{i} h^{i j} N_{j}, \quad g_{0 i}=N_{i}, \quad g_{i j}=h_{i j}, \quad \operatorname{det} g=-N^{2} \operatorname{det} h
$$

with inverse

$$
g^{00}=-\frac{1}{N^{2}}, \quad g^{0 i}=\frac{N^{i}}{N}, \quad g^{i j}=h^{i j}-\frac{N^{i} N^{j}}{N^{2}} .
$$

Now we are ready to formulate the Hamiltonian description of point particle in the background with broken Lorenz invariance. This problem was studied in [72, 73, 74, 75, 76, 77] where it was argued that Hamiltonian constraint should have the form

$$
H_{T}=-\frac{1}{N^{2}}\left(p_{t}-N^{i} p_{i}\right)^{2}+h\left(p^{2}\right) \approx 0, \quad p^{2}=p_{i} p_{j} h^{i j}
$$

so that the Hamiltonian is given as

$$
H=\lambda(t) H_{T},
$$

where $\lambda(t)$ is the Lagrange multiplier that express the fact that the Hamiltonian is proportional to the first class constraint (2.3). This form of Hamiltonian constraint reflects the non-relativistic nature of the Hořava-Lifshitz gravities where generally $h(A)=A^{n}$ for some positive $n$. In particular, the analysis of the dynamics of the probe particle that is governed by this Hamiltonian brought many interesting results. For example, it was argued that particles with such a form of the Hamiltonian does not feel the presence of the horizon in the background of black hole ${ }^{2}$.

Now we determine Lagrangian corresponding to the Hamiltonian (2.4). It is useful to introduce two non-dynamical modes $A, B$ together with their conjugate momenta $P_{A}, P_{B}$ and rewrite the Hamiltonian constraint in the form

$$
H_{T}=-\frac{1}{N^{2}}\left(p_{t}-N^{i} p_{i}\right)^{2}+B\left(p^{2}-A\right)+h(A)
$$

so that the Hamiltonian takes the form

$$
H=\lambda(\tau) H_{T}+v_{A} P_{A}+v_{B} P_{B}
$$

where $v_{A}, v_{B}$ are Lagrange multipliers that ensure that $P_{A}, P_{B}$ are primary constraints of the theory

$$
P_{A} \approx 0, \quad P_{B} \approx 0
$$

In fact, the consistency of these constraints with the time evolution of the system implies two secondary constraints $G_{A}, G_{B}$. Explicitly

$$
\frac{d P_{A}}{d \tau}=\left\{P_{A}, H\right\}=\lambda(\tau)\left(B-h^{\prime}(A)\right)=\lambda(\tau) G_{A} \approx 0, \quad h^{\prime}(A) \equiv \frac{d h(A)}{d A}
$$

and

$$
\frac{d P_{B}}{d \tau}=\left\{P_{B}, H\right\}=-\lambda(\tau)\left(p^{2}-A\right)=\lambda(\tau) G_{B} \approx 0 .
$$

\footnotetext{
${ }^{2}$ For further details we recommend the original papers $[72,73,74,75,76,77$.
} 
It can be shown that the collection of the constraints $\left(P_{A}, P_{B}, G_{A}, G_{B}\right)$ form the set of the second class constraints that can be explicitly solved as $p^{2}=A$ and $B=h^{\prime}(A)$. Then inserting these results into 2.5$)$ we recovery the original Hamiltonian constraint (2.3).

Now with the help of the extended Hamiltonian it is easy to find the corresponding Lagrangian since

$$
\begin{aligned}
\frac{d t}{d \tau} & =\{t, H\}=-\frac{2 \lambda}{N^{2}}\left(p_{t}-N^{i} p_{i}\right), \\
\frac{d x^{i}}{d \tau} & =\left\{x^{i}, H\right\}=\frac{2 \lambda N^{i}}{N^{2}}\left(p_{t}-N^{j} p_{j}\right)+2 \lambda B h^{i j} p_{j}, \\
\frac{d A}{d \tau} & =\{A, H\}=v_{A}, \quad \frac{d B}{d \tau}=\{B, H\}=v_{B} .
\end{aligned}
$$

Then it is easy to find corresponding Lagrangian

$$
\begin{aligned}
L & =p_{M} \frac{d x^{M}}{d \tau}+P_{A} \frac{d A}{d \tau}+P_{B} \frac{d B}{d \tau}-H= \\
& =-\frac{N^{2}}{4 \lambda}\left(\frac{d t}{d \tau}\right)^{2}+\frac{1}{4 B \lambda} V^{i} V^{j} h_{i j}+\lambda B A-\lambda h(A),
\end{aligned}
$$

where

$$
V^{i}=\frac{d x^{i}}{d \tau}-N^{i} \frac{d t}{d \tau} .
$$

As the final step we integrate out $A, B$ from (2.11). Explicitly, the variation of (2.11) with respect to $A$ and $B$ implies the equation of motion

$$
\begin{aligned}
& B-h^{\prime}(A)=0, \\
& -\frac{1}{4 B^{2} \lambda} V^{i} V^{j} h_{i j}+A \lambda=0 .
\end{aligned}
$$

The first equation in (2.13) implies $B=h^{\prime}(A)$. Inserting this result into the second equation in (2.13) we obtain an algebraic equation for $A$

$$
-\frac{1}{4 h^{\prime 2}(A) \lambda^{2}} V^{i} V^{j} h_{i j}+A=0 .
$$

For given function $h(A)$ this equation can be solved for $A$

$$
A=\Psi\left(\frac{1}{4 \lambda^{2}} V^{i} V^{j} h_{i j}\right) .
$$

Then inserting (2.13), (2.14) and (2.15) into (2.11) we find the Lagrangian in the form

$$
L=\lambda\left[-\frac{N^{2}}{4 \lambda^{2}}\left(\frac{d t}{d \tau}\right)^{2}+\frac{2}{h^{\prime}\left(\Psi\left(\frac{1}{4 \lambda^{2}} V^{i} V^{j} h_{i j}\right)\right)} \frac{1}{4 \lambda^{2}} V^{i} V^{j} h_{i j}-h\left(\Psi\left(\frac{1}{4 \lambda^{2}} V^{i} V^{j} h_{i j}\right)\right)\right] .
$$


Now we show that the action $S=\int d \tau L$ with $L$ given in (2.16) is invariant under the world-line diffeomorphism

$$
\tau^{\prime}=\tau^{\prime}(\tau)
$$

As the first step we postulate that $\lambda(\tau)$ transforms under (2.17) as

$$
\lambda^{\prime}\left(\tau^{\prime}\right)=\lambda(\tau) \frac{d \tau}{d \tau^{\prime}}
$$

so that $d \tau \lambda(\tau)$ is invariant under (2.17). Further we demand that $t, x^{i}$ behave as scalars under 2.17)

$$
t^{\prime}\left(\tau^{\prime}\right)=t(\tau), \quad x^{\prime i}\left(\tau^{\prime}\right)=x^{i}(\tau)
$$

Consequently we find

$$
V^{\prime i}\left(\tau^{\prime}\right)=V^{i}(\tau) \frac{d \tau}{d \tau^{\prime}}, \quad \frac{d t^{\prime}\left(\tau^{\prime}\right)}{d \tau^{\prime}}=\frac{d t(\tau)}{d \tau} \frac{d \tau}{d \tau^{\prime}}
$$

Using these results we immediately see that $\frac{1}{\lambda^{2}} V^{i} V^{j} h_{i j}$ and $\frac{1}{\lambda^{2}}\left(\frac{d t}{d \tau}\right)^{2}$ are invariant under (2.17). In summary we see that the action is invariant under (2.17).

We conclude this section we some examples of the function $h=h(A)$. As the first one we consider $h=\frac{1}{2 n} A^{n}$. For this function the equation (2.14) has the solution

$$
A=\left(\frac{1}{\lambda^{2}} V^{i} V^{j} h_{i j}\right)^{\frac{1}{2 n-1}}
$$

and consequently the Lagrangian takes the form

$$
L=\lambda\left[-\frac{N^{2}}{4 \lambda^{2}}\left(\frac{d t}{d \tau}\right)^{2}+\left(1-\frac{1}{2 n}\right)\left(\frac{1}{\lambda^{2}} V V^{j} h_{i j}\right)^{\frac{n}{2 n-1}}\right] .
$$

This is the Lagrangian studied in 72, 73, 74, 75, 76, 77.

As the second example we consider the function $h=\sqrt{1+A}$. For this function the equation (2.14) has the solution

$$
A=\frac{V^{i} V^{j} h_{i j}}{\lambda^{2}\left(1-\frac{1}{\lambda^{2}} V^{i} V^{j} h_{i j}\right)}
$$

and consequently the Lagrangian takes the form

$$
L=\lambda\left[-\frac{N^{2}}{4 \lambda^{2}}\left(\frac{d t}{d \tau}\right)^{2}-\sqrt{1-\frac{1}{\lambda^{2}} V^{i} V^{j} h_{i j}}\right] .
$$

\section{Lorentz Breaking String Theory}

In this section we generalize the analysis presented in the previous section to the case of string probe in Hořava-Lifshitz background when we study properties of the string with the new form of the Hamiltonian constraint that generalizes the point particle Hamiltonian constraint studied in the previous section. In fact, since it is not know how or whether it is 
possible to formulate Hořava-Lifshitz gravities in the framework of string theories we mean that it is legitime to consider string probe in given background with Hamiltonian constraint that breaks the Lorentz invariance of the target space-time which is the characteristic property of Hořava-Lifshitz background.

As in case of point particle LBS theory is defined using the Hamiltonian formalism that is based on the Hamiltonian analysis of relativistic string. For that reason we now review the Hamiltonian formulation of the Polyakov string action in general background.

\subsection{Hamiltonian formulation of Polyakov Action}

Let us consider the Polyakov action in general background

$$
S=-\frac{1}{4 \pi \alpha^{\prime}} \int d^{2} \sigma \sqrt{-\gamma} \gamma^{\alpha \beta} g_{M N} \partial_{\alpha} x^{M} \partial_{\beta} x^{N}
$$

where $\gamma_{\alpha \beta}$ is a two-dimensional world-sheet metric and $\sigma^{\alpha}, \alpha, \beta=0,1, \sigma^{0}=\tau, \sigma^{1}=\sigma$

are world-sheet coordinates. Further, $x^{M}, M, N=0, \ldots, D$ are modes that parameterize the embedding of the string into target space-time with the background metric $g_{M N}$.

In order to formulate the Hamiltonian formalism from the action (3.1) it is convenient to use $1+1$ formalism for the world-sheet metric

$$
\gamma_{\alpha \beta}=\left(\begin{array}{cc}
-n_{\tau}^{2}+\frac{1}{\omega} n_{\sigma}^{2} & n_{\sigma} \\
n_{\sigma} & \omega
\end{array}\right)
$$

where $n_{\tau}$ is world-sheet lapse, $n_{\sigma}$ is world-sheet shift and $\omega$ is spatial part of world-sheet metric. Then it is easy to see that

$$
\operatorname{det} \gamma=-n_{\tau}^{2} \omega, \quad \gamma^{\alpha \beta}=\left(\begin{array}{cc}
-\frac{1}{n_{\tau}^{2}} & \frac{n^{\sigma}}{n_{\tau}^{2}} \\
\frac{n^{\sigma}}{n_{\tau}^{2}} & \frac{1}{\omega}-\frac{n^{\sigma} n^{\sigma}}{n_{\tau}^{2}}
\end{array}\right),
$$

where we defined

$$
n^{\sigma} \equiv \frac{n_{\sigma}}{\omega}
$$

With this form of the world-sheet metric the action (3.1) takes the form

$$
S=\frac{1}{4 \pi \alpha^{\prime}} \int d^{2} \sigma n_{\tau} \sqrt{\omega}\left(g_{M N} \nabla_{\tau} x^{M} \nabla_{\tau} x^{N}-\frac{1}{\omega} g_{M N} \partial_{\sigma} x^{M} \partial_{\sigma} x^{N}\right)
$$

where

$$
\nabla_{\tau} x^{M}=\frac{1}{n_{\tau}}\left(\partial_{\tau} x^{M}-n^{\sigma} \partial_{\sigma} x^{M}\right) .
$$

We introduce the momenta $\pi^{\tau}, \pi^{\sigma}, \pi^{\omega}$ conjugate to $n_{\tau}, n_{\sigma}$ and $\omega$ that have non-zero Poisson brackets

$$
\left\{n_{\tau}(\sigma), \pi^{\tau}\left(\sigma^{\prime}\right)\right\}=\delta\left(\sigma-\sigma^{\prime}\right), \quad\left\{n_{\sigma}(\sigma), \pi^{\sigma}\left(\sigma^{\prime}\right)\right\}=\delta\left(\sigma-\sigma^{\prime}\right), \quad\left\{\omega(\sigma), \pi^{\omega}\left(\sigma^{\prime}\right)\right\}=\delta\left(\sigma-\sigma^{\prime}\right) .
$$


Since there are no time derivatives of world-sheet metric $\gamma$ in (3.5) the conjugate momenta are primary constraints of theory

$$
\pi^{\tau}=\frac{\delta S}{\delta \partial_{\tau} n_{\tau}} \approx 0, \quad \pi^{\sigma}=\frac{\delta S}{\delta \partial_{\tau} n_{\sigma}} \approx 0, \quad \pi^{\omega}=\frac{\delta S}{\delta \partial_{\tau} \omega} \approx 0
$$

As the next step we introduce the momenta $p_{M}$ conjugate to $x^{M}$

$$
p_{M}=\frac{\delta S}{\delta \partial_{\tau} x^{M}}=\frac{1}{2 \pi \alpha^{\prime}} \sqrt{\omega} g_{M N} \nabla_{\tau} x^{N}
$$

with standard Poisson brackets

$$
\left\{x^{M}(\sigma), p_{N}\left(\sigma^{\prime}\right)\right\}=\delta_{N}^{M} \delta\left(\sigma-\sigma^{\prime}\right) .
$$

Then it is easy to find the Hamiltonian density

$$
\mathcal{H}=\partial_{\tau} x^{N} p_{M}-\mathcal{L}=n_{\tau} \mathcal{H}_{\tau}+n^{\sigma} \mathcal{H}_{\sigma}
$$

where

$$
\begin{aligned}
\mathcal{H}_{T} & =\frac{1}{4 \pi \alpha^{\prime} \sqrt{\omega}}\left(\left(2 \pi \alpha^{\prime}\right)^{2} p_{M} g^{M N} p_{N}+\partial_{\sigma} x^{M} g_{M N} \partial_{\sigma} x^{N}\right) \\
\mathcal{H}_{S} & =\partial_{\sigma} x^{N} p_{N}
\end{aligned}
$$

Using the Hamiltonian (3.11) we easily determine the time evolution of the primary constraints as

$$
\begin{aligned}
& \partial_{\tau} \pi_{\tau}=\left\{\pi_{\tau}, H\right\}=-\mathcal{H}_{\tau} \\
& \partial_{\tau} \pi_{\omega}=\left\{\pi_{\omega}, H\right\}=\frac{n_{\tau}}{2 \omega} \mathcal{H}_{\tau}+\frac{1}{\omega^{2}} n_{\sigma} \mathcal{H}_{\sigma} \\
& \partial_{\tau} \pi_{\sigma}=\left\{\pi_{\sigma}, H\right\}=-\mathcal{H}_{\sigma} .
\end{aligned}
$$

Since the constraints (3.8) have to be preserved during the time evolution of the system the equations above imply an existence of the secondary constraints

$$
\mathcal{H}_{\tau} \approx 0, \quad \mathcal{H}_{\sigma} \approx 0
$$

The existence of these constraints will be the starting point for the construction of Lorentz breaking string theories.

\subsection{Lorentz Breaking String Action}

The construction of Lorentz breaking string theories is based on the generalization of the point particle analysis reviewed in the first section. Due to the fact that the world-sheet modes depend on $\sigma$ there are several possibilities how to do it. We consider the case when 
we presume that $p_{t}$ and $t$ are functions of $\tau$ only and suggest following form of constraints (3.12)

$$
\begin{aligned}
& \mathcal{H}_{\tau}=-\frac{\pi \alpha^{\prime}}{N^{2} \sqrt{\omega}}\left(p_{t}-N^{i} p_{i}\right)^{2}+\sqrt{\omega} F\left(\frac{1}{\omega}\left[\pi \alpha^{\prime} p_{i} h^{i j} p_{j}+\frac{1}{4 \pi \alpha^{\prime}} \partial_{\sigma} x^{i} \partial_{\sigma} x^{j} h_{i j}\right]\right), \\
& \mathcal{H}_{\sigma}=p_{i} \partial_{\sigma} x^{i}
\end{aligned}
$$

where $F$ is an arbitrary function. Our goal is to study properties of string theory with this form of the Hamiltonian constraint $\mathcal{H}_{\tau}$.

As the first step we find the Lagrangian formulation of given theory. Following analysis performed in section (2) we introduce two non-dynamical fields $A, B$ in order to rewrite the Hamiltonian constraint into the form

$$
\begin{aligned}
\mathcal{H}_{\tau} & =-\frac{\pi \alpha^{\prime}}{N^{2} \sqrt{\omega}}\left(p_{t}-N^{i} p_{i}\right)^{2}+ \\
& +B\left(\frac{1}{\omega}\left[\pi \alpha^{\prime} p_{i} h^{i j} p_{j}+\frac{1}{4 \pi \alpha^{\prime}} \partial_{\sigma} x^{i} \partial_{\sigma} x^{j} h_{i j}\right]-A\right)+\sqrt{\omega} F(A)+v_{A} P_{A}+v_{B} P_{B},
\end{aligned}
$$

where $v_{A}, v_{B}$ are Lagrange multipliers that ensure that the momenta $P_{A}, P_{B}$ vanish. Then from the Hamiltonian

$$
H=\int d \sigma\left(n_{\tau} \mathcal{H}_{\tau}+n^{\sigma} \mathcal{H}_{\sigma}\right)
$$

where $\mathcal{H}_{\tau}$ and $\mathcal{H}_{\sigma}$ are given in (3.16) we derive following equations of motion for $t, x^{i}, A, B$

$$
\begin{aligned}
\partial_{\tau} t & =\{t, H\}=-\frac{2 \pi \alpha^{\prime}}{N^{2} \sqrt{\omega}} n_{\tau}\left(p_{t}-N^{i} p_{i}\right), \\
\partial_{\tau} x^{i} & =\left\{x^{i}, H\right\}=2 \pi \alpha^{\prime} \frac{n_{\tau}}{\sqrt{\omega}} \frac{N^{i}}{N^{2}}\left(p_{t}-N^{j} p_{j}\right)+2 \pi \alpha^{\prime} \frac{n_{\tau}}{\omega} B h^{i j} p_{j}+\frac{1}{\omega} n_{\sigma} \partial_{\sigma} x^{i}, \\
\partial_{\tau} A & =\{A, H\}=n_{\tau} v_{A}, \quad \partial_{\tau} B=\{B, H\}=n_{\tau} v_{B} .
\end{aligned}
$$

Then it is easy to find corresponding Lagrangian density

$$
\begin{aligned}
L & =\partial_{\tau} x^{M} p_{M}+\partial_{\tau} A P_{A}+\partial_{\tau} B P_{B}-H= \\
& =-\frac{N^{2} \sqrt{\omega} n_{\tau}}{4 \pi \alpha^{\prime} n_{\tau}^{2}}\left(\partial_{\tau} t\right)^{2}+\frac{n_{\tau} \omega}{4 \pi \alpha^{\prime}} \frac{1}{B n_{\tau}^{2}} V^{i} h_{i j} V^{j}- \\
& -n_{\tau} B\left(\frac{1}{4 \pi \alpha^{\prime} \omega} \partial_{\sigma} x^{i} \partial_{\sigma} x^{j} h_{i j}-A\right)-n_{\tau} \sqrt{\omega} F(A),
\end{aligned}
$$

where

$$
V^{i} \equiv \partial_{\tau} x^{i}+\partial_{\tau} t N^{i}-n^{\sigma} \partial_{\sigma} x^{i}
$$


Finally we integrate out non-dynamical fields $A$ and $B$ by solving their equations of motion. The equation of motion for $A$ implies

$$
B-\sqrt{\omega} F^{\prime}(A)=0
$$

while the equation of motion for $B$ gives

$$
-\frac{1}{4 \pi \alpha^{\prime}} \frac{\omega}{n_{\tau}^{2} B^{2}} V^{i} h_{i j} V^{j}-n_{\tau}\left(\frac{1}{4 \pi \alpha \omega} \partial_{\sigma} x^{i} \partial_{\sigma} x^{j} h_{i j}-A\right)=0 .
$$

For known $F(A)$ these equations can be solved for $A$

$$
A=\Psi\left(\frac{1}{4 \pi \alpha^{\prime}} \frac{1}{n_{\tau}^{2}} V^{i} h_{i j} V^{j}, \frac{1}{4 \pi \alpha^{\prime} \omega} \partial_{\sigma} x^{i} \partial_{\sigma} x^{j} h_{i j}\right) .
$$

As a result we find that the Lagrangian density in the form

$$
\mathcal{L}=\sqrt{\omega} n_{\tau}\left[-\frac{N^{2}}{4 \pi \alpha^{\prime} n_{\tau}^{2}}\left(\partial_{\tau} t\right)^{2}+\frac{1}{2 \pi \alpha^{\prime}} \frac{1}{F^{\prime}(\Psi) n_{\tau}^{2}} V^{i} h_{i j} V^{j}-F(\Psi)\right] .
$$

Clearly this form of the Lagrangian is completely different from the Polyakov Lagrangian. It is very interesting to study the consequence of the breaking of the target space covariance. In particular we would like to identify symmetries of the Lagrangian density (3.24).

\subsection{Symmetries of the action}

In this section we study the symmetries of the action $S=\int d \tau d \sigma \mathcal{L}$ where $\mathcal{L}$ is given in (3.24). We begin with the discussion of the global symmetries of the action from the point of view of the string world-sheet theory. These transformations correspond to the target space foliation preserving diffeomorphism [2]

$$
\begin{aligned}
t^{\prime}(\tau) & =t(\tau)+f(t(\tau)), \\
x^{\prime i}(\tau, \sigma) & =x^{i}(\tau, \sigma)+\xi^{i}\left(t(\tau), x^{i}(\tau, \sigma)\right)
\end{aligned}
$$

so that

$$
\begin{aligned}
\partial_{\tau} t^{\prime} & =\partial_{\tau} t+\dot{f} \partial_{\tau} t \\
\partial_{\tau} x^{i} & =\partial_{\tau} x^{i}+\partial_{j} \xi^{i} \partial_{\tau} x^{j}+\dot{\xi}^{i} \partial_{\tau} t \\
\partial_{\sigma} x^{i} & =\partial_{\sigma} x^{i}+\partial_{j} \xi^{i} \partial_{\sigma} x^{j}
\end{aligned}
$$

where

$$
\dot{F}=\frac{d F}{d t}, \quad \partial_{i} F=\frac{\partial F}{\partial x^{i}}
$$

for any $F$. Note that under these transformations the metric components transform as

$$
\begin{aligned}
N_{i}^{\prime}\left(t^{\prime}, \mathbf{x}^{\prime}\right) & =N_{i}(t, \mathbf{x})-N_{i}(t, \mathbf{x}) \dot{f}(t)-N_{j}(t, \mathbf{x}) \partial_{i} \zeta^{j}(t, \mathbf{x})-g_{i j}(t, \mathbf{x}) \dot{\zeta}^{j}(t, \mathbf{x}) \\
N^{\prime}\left(t^{\prime}\right) & =N(t)-N(t) \dot{f}(t) \\
g_{i j}^{\prime}\left(t^{\prime}, \mathbf{x}^{\prime}\right) & =g_{i j}(t, \mathbf{x})-g_{i l}(t, \mathbf{x}) \partial_{j} \zeta^{l}(t, \mathbf{x})-\partial_{i} \zeta^{k}(t, \mathbf{x}) g_{k j}(t, \mathbf{x})
\end{aligned}
$$


Then it is easy to see that

$$
\begin{aligned}
N^{\prime 2}\left(t^{\prime}\right)\left(\partial_{\tau} t^{\prime}\right)^{2} & =N^{2}(t)\left(\partial_{\tau} t\right)^{2}, \\
\left(V^{i} h_{i j} V^{j}\right)^{\prime} & =V^{i} h_{i j} V^{j} \\
\left(\partial_{\sigma} x^{i} h_{i j} \partial_{\sigma} x^{j}\right)^{\prime} & =\partial_{\sigma} x^{i} h_{i j} \partial_{\sigma} x^{j},
\end{aligned}
$$

using also the fact that $V^{i}$ transforms under (3.25) as

$$
V^{i}=V^{i}+\partial_{j} \xi^{i} V^{j}
$$

Collecting all these results we find that the Lagrangian density (3.24) is invariant under (3.25).

Let us now discuss the local symmetries of the action. We argue that this action is invariant under world-sheet foliation preserving diffeomorphism that are generated by infinitesimal transformations

$$
\delta \sigma \equiv \sigma^{\prime}-\sigma=\zeta(\tau, \sigma), \quad \delta \tau \equiv \tau^{\prime}-\tau=f(\tau) .
$$

In the same way as in [2] we find that the world-sheet metric components transform under (3.31) as

$$
\begin{aligned}
n_{\sigma}^{\prime}\left(\tau^{\prime}, \sigma^{\prime}\right) & =n_{\sigma}(\tau, \sigma)-n_{\sigma}(\tau, \sigma) \partial_{\sigma} \epsilon(\tau, \sigma)-\partial_{\tau} f(\tau) n_{\sigma}(\tau, \sigma)-\partial_{\tau} \epsilon(\tau, \sigma) \omega(\tau, \sigma), \\
n_{\tau}^{\prime}\left(\tau^{\prime}, \sigma^{\prime}\right) & =n_{\tau}(\tau, \sigma)-n_{\tau}(\tau, \sigma) \partial_{\tau} f(\tau) \\
\omega^{\prime}\left(\tau^{\prime}, \sigma^{\prime}\right) & =\omega(\tau, \sigma)-2 \partial_{\sigma} \epsilon(\tau, \sigma) \omega(\tau, \sigma) \\
n^{\prime \sigma}\left(\tau^{\prime}, \sigma^{\prime}\right) & \equiv \frac{n_{\sigma}^{\prime}\left(\tau^{\prime}, \sigma^{\prime}\right)}{\omega^{\prime}\left(\tau^{\prime}, \sigma^{\prime}\right)}=n^{\sigma}(\tau, \sigma)+n^{\sigma}(\tau, \sigma) \partial_{\sigma} \epsilon(\tau, \sigma)-n^{\sigma}(\tau, \sigma) \partial_{\tau} f(\tau)-\partial_{\tau} \epsilon(\tau, \sigma) .
\end{aligned}
$$

Then it is easy to see that

$$
d \tau^{\prime} d \sigma^{\prime} n_{\tau}^{\prime} \sqrt{\omega^{\prime}}=d \tau d \sigma n_{\tau} \sqrt{\omega}
$$

Clearly, the world-sheet modes $x^{M}$ transform as scalars under (3.31)

$$
x^{M}\left(\tau^{\prime}, \sigma^{\prime}\right)=x^{M}(\tau, \sigma) .
$$

Then we easily show that

$$
\frac{1}{n_{\tau}^{\prime 2}\left(\tau^{\prime}\right)}\left(\partial_{\tau^{\prime}} t^{\prime}\left(\tau^{\prime}\right)\right)^{2}=\frac{1}{n_{\tau}^{2}(\tau)}\left(\partial_{\tau} t(\tau)\right)^{2}
$$

and

$$
\left(\frac{1}{n_{\tau}^{2}} V^{i} h_{i j} V^{j}\right)^{\prime}\left(\tau^{\prime}, \sigma^{\prime}\right)=\frac{1}{n_{\tau}^{2}} V^{i} h_{i j} V^{j}(\tau, \sigma)
$$


using the fact that

$$
V^{\prime i}\left(\tau^{\prime}, \sigma^{\prime}\right)=V^{i}(\tau, \sigma)-V^{i}(\tau, \sigma) \partial_{\tau} f(\tau)
$$

In the same way we can show that

$$
\left(\frac{1}{\omega} \partial_{\sigma} x^{i} \partial_{\sigma} x^{j} h_{i j}\right)^{\prime}\left(\tau^{\prime}, \sigma^{\prime}\right)=\frac{1}{\omega} \partial_{\sigma} x^{i} \partial_{\sigma} x^{j} h_{i j}(\tau, \sigma) .
$$

Again, collecting all these results we find that the action $S=\int d \tau d \sigma \mathcal{L}$ with $\mathcal{L}$ given in (3.24) is invariant under world-sheet foliation preserving diffeomorphism. In other words, the Lorentz braking form of the Hamiltonian constraint is consistent with the world-sheet theory where the full diffeomorphism invariance is reduced. It is important to stress that at this moment we still presume the general dependence of $n_{\tau}$ on $\tau$ and $\sigma$. In other words, we formulate theory without projectability condition imposed. We discuss the problem of projectability in the next section.

Now we give explicit examples of the function $F$. The first one is the polynomial function $F=\frac{1}{n} A^{n}$ known from the point particle analysis in the Horava-Lifshitz background. For this function $B=\sqrt{\omega} A^{n-1}$ and then the equation (3.22) leads to polynomial equation for $A$ where it is difficult to find their roots analytically. Clearly the resulting Lagrangian density is very complicated and it is hardly to see how such a form of the Lagrangian could be deduced from the first principles.

As the second example we consider the function $F(A)=\sqrt{1+A}$. For this form of the function it is possible to find solution (3.22). On the other hand this function does not have a support from the study of the probe in Hořava-Lifshitz gravity. Explicitly, the equation (3.21) implies $B=\frac{1}{2 \sqrt{1+A}} \sqrt{\omega}$ and then the equation (3.22) has solution

$$
A=\frac{1}{1-\frac{4}{4 \pi \alpha^{\prime}} \frac{V^{i} h_{i j} V^{j}}{n_{\tau}^{2}}}\left(\frac{1}{4 \pi \alpha^{\prime} \omega} \partial_{\sigma} x^{i} h_{i j} \partial_{\sigma} x^{j}+\frac{4}{4 \pi \alpha^{\prime}} \frac{1}{n_{\tau}^{2}} V^{i} h_{i j} V^{j}\right)
$$

Then it is easy to find the Lagrangian density in the form

$$
\mathcal{L}=\sqrt{\omega} n_{\tau}\left[-\frac{N^{2}}{4 \pi \alpha^{\prime} n_{\tau}^{2}}\left(\partial_{\tau} t\right)^{2}-\sqrt{\frac{1}{4 \pi \alpha^{\prime} \omega} \partial_{\sigma} x^{i} \partial_{\sigma} x^{j} h_{i j}+\frac{1}{\pi \alpha^{\prime}} \frac{1}{n_{\tau}^{2}} V^{i} h_{i j} V^{j}} \sqrt{1-\frac{1}{\pi \alpha^{\prime}} \frac{1}{n_{\tau}^{2}} V^{i} h_{i j} V^{j}}\right]
$$

We conclude this section with the second example of the Lorentz breaking Hamiltonian constraint

$$
\mathcal{H}_{\tau}=-\frac{\pi \alpha^{\prime}}{N^{2} \sqrt{\omega}}\left(p_{t}-N^{i} p_{i}\right)^{2}++\sqrt{\omega} F\left(\frac{1}{\omega} \pi \alpha^{\prime} p_{i} h^{i j} p_{j}\right)+\sqrt{\omega} G\left(\frac{1}{4 \pi \alpha^{\prime} \omega} \partial_{\sigma} x^{i} h_{i j} \partial_{\sigma} x^{j}\right)
$$


where $F$ and $G$ are arbitrary functions. Then, following the same analysis as above we find the Lagrangian density in the form

$\mathcal{L}=n_{\tau} \sqrt{\omega}\left[-\frac{N^{2}}{4 \pi \alpha^{\prime} n_{\tau}^{2}}\left(\partial_{\tau} t\right)^{2}+\frac{1}{2 \pi \alpha^{\prime}} \frac{1}{F^{\prime}(\Psi) n_{\tau}^{2}} V^{i} h_{i j} V^{j}-G\left(\frac{1}{4 \pi \alpha^{\prime} \omega} \partial_{\sigma} x^{i} \partial_{\sigma} x^{j} h_{i j}\right)-F(\Psi)\right]$.

Clearly this Lagrangian density is invariant under the same set of symmetries as (3.24) and hence can be considered as another example of Lorentz breaking string theory. Note that for this form of the Hamiltonian constraint it is possible to find explicit form of the Lagrangian density even in case of the function $F=\frac{1}{n} A^{n}$. In fact, it is to see that $A$ is equal to

$$
A=\left(\frac{n}{4 \pi \alpha^{\prime}} \frac{V^{i} h_{i j} V^{j}}{n_{\tau}^{2}}\right)^{\frac{1}{2 n-1}}
$$

and consequently the Lagrangian density takes the form

$\mathcal{L}=n_{\tau} \sqrt{\omega}\left[-\frac{N^{2}}{4 \pi \alpha^{\prime} n_{\tau}^{2}}\left(\partial_{\tau} t\right)^{2}+n^{\frac{1-n}{2 n-1}}\left(\frac{1}{4 \pi \alpha^{\prime} n_{\tau}^{2}} V^{i} h_{i j} V^{j}\right)^{\frac{n}{2 n-1}}-G\left(\frac{1}{4 \pi \alpha^{\prime} \omega} \partial_{\sigma} x^{i} \partial_{\sigma} x^{j} h_{i j}\right)\right]$.

This Lagrangian density takes similar form as the particle Lagrangian studied in the second section. In other words, the extension of the Lorentz breaking Hamiltonian constraint from the point particle to string is not unique that should be compared with relatively straightforward step from relativistic particle to relativistic string.

\section{Hamiltonian Formalism of Non-Relativistic String Theory Revised}

In the previous section we found new class of non-relativistic string theories when we presumed Hamiltonian constraint that breaks Lorentz invariance of the target space. However it is important to stress that it is not possible to change the Hamiltonian constraint freely without further checking of the consistency of given theory. In fact, let us again consider the Hamiltonian constraint

$$
\mathcal{H}_{\tau}=-\frac{\pi \alpha^{\prime}}{N^{2} \sqrt{\omega}}\left(p_{t}-N^{i} p_{i}\right)^{2}+\sqrt{\omega} F\left(\frac{1}{\omega}\left[\pi \alpha^{\prime} p_{i} h^{i j} p_{j}+\frac{1}{4 \pi \alpha^{\prime}} \partial_{\sigma} x^{i} \partial_{\sigma} x^{j} h_{i j}\right]\right) .
$$

As follows from (4.1) the Hamiltonian constraint depends on $\omega$ in non-trivial way. However this fact suggests that it is not useful to impose the primary constraint $\pi^{\omega} \approx 0$ since then the requirement of the consistency of this constraint with the time evolution of the system implies additional constraints. Then in order to avoid imposing additional constraint on the system we demand that $\omega$ is a dynamical mode with kinetic term in the action. The simplest possibility is to add to the string action following kinetic term

$$
S_{\omega}^{K}=\frac{1}{2} \int d \tau d \sigma n_{\tau} \sqrt{\omega} K_{\sigma} \frac{1}{\omega^{2}} K_{\sigma}
$$


where

$$
\begin{aligned}
K_{\sigma} & =\frac{1}{n_{\tau}}\left(\partial_{\tau} \omega-2 \nabla_{\sigma} n_{\sigma}\right) \\
\nabla n_{\sigma} & =\partial_{\sigma} n_{\sigma}-\Gamma n_{\sigma}, \quad \Gamma=\frac{1}{2 \omega} \partial_{\sigma} \omega .
\end{aligned}
$$

Note that $\Gamma$ transforms under the world-sheet foliation preserving diffeomorphism (3.31) as

$$
\Gamma^{\prime}\left(\tau^{\prime}, \sigma^{\prime}\right)=\Gamma(\tau, \sigma)-\Gamma(\tau, \sigma) \partial_{\sigma} \epsilon(\tau, \sigma)-\partial_{\sigma}^{2} \epsilon(\tau, \sigma)
$$

so that $\nabla_{\sigma} n_{\sigma}$ transforms as

$$
\left(\nabla_{\sigma} n_{\sigma}\right)^{\prime}=\nabla_{\sigma} n_{\sigma}-2 \nabla_{\sigma} n_{\sigma} \partial_{\sigma} \epsilon-\nabla_{\sigma} n_{\sigma} \partial_{\tau} f-\partial_{\sigma} \partial_{\tau} \epsilon \omega-\frac{1}{2} \partial_{\tau} \epsilon \partial_{\sigma} \omega
$$

Then after some algebra we find that $K_{\sigma}$ transforms as

$$
K_{\sigma}^{\prime}\left(\tau^{\prime}, \sigma^{\prime}\right)=K_{\sigma}(\tau, \sigma)-2 K_{\sigma}(\tau, \sigma) \partial_{\sigma} \epsilon(\tau, \sigma)
$$

This result implies that $S_{\omega}^{K}$ is manifestly invariant under (3.31). We should also stress that this choice of the kinetic term is the minimal one. For example, we could in principle include the term $\frac{1}{\omega^{2}} K_{\sigma}^{2}$ into the factor of the function $F$. For simplicity we consider the first option and we find

$$
\pi^{\omega}=\frac{\delta S_{\omega}^{K}}{\partial_{\tau} \omega}=\sqrt{\omega} n_{\tau} \frac{1}{\omega^{2} n_{\tau}} K_{\sigma}
$$

Then it is easy to see that the total Hamiltonian takes the form

$$
\mathcal{H}=n_{\tau} \mathcal{H}_{\tau}+n^{\sigma} \mathcal{H}_{\sigma}+\lambda_{\tau} \pi^{\tau}+\lambda_{\sigma} \pi^{\sigma}
$$

where

$$
\begin{aligned}
\mathcal{H}_{\tau} & =-\frac{\pi \alpha^{\prime}}{N^{2} \sqrt{\omega}}\left(p_{t}-N^{i} p_{i}\right)^{2}+\frac{1}{2 \sqrt{\omega}} \pi^{\omega} \omega^{2} \pi^{\omega}+ \\
& +\sqrt{\omega} F\left(\frac{1}{\omega}\left[\pi \alpha^{\prime} p_{i} h^{i j} p_{j}+\frac{1}{4 \pi \alpha^{\prime}} \partial_{\sigma} x^{i} \partial_{\sigma} x^{j} h_{i j}\right]\right), \\
\mathcal{H}_{\sigma} & =p_{i} \partial_{\sigma} x^{i}-2 \omega \nabla_{\sigma} \pi^{\sigma} .
\end{aligned}
$$

In other words, the LBS theory is characterized by two primary constraints $\pi^{\tau} \approx 0, \pi^{\sigma} \approx 0$ together with two secondary ones $\mathcal{H}_{\tau} \approx 0, \mathcal{H}_{\sigma} \approx 0$ where we still presume that $n_{\tau}$ depends on $\tau$ and $\sigma$. 
As the next step in the analysis of the consistency of given theory we calculate the algebra of constraints and we demand that it is closed. Alternatively, we should check that the consistency of these constraints with the time evolution of the system either does not impose additional constraints on the theory or they are not the second class constraints which would signal the pathological behavior of given theory [57].

In order to calculate the algebra of constraints we introduce their smeared form

$$
\mathbf{T}_{S}(\xi)=\int d \sigma \xi(\tau, \sigma) \mathcal{H}_{\sigma}, \quad \mathbf{T}_{T}(f)=\int d \sigma f(\tau, \sigma) \mathcal{H}_{\tau}
$$

Then using the canonical Poisson brackets

$$
\left\{x^{M}(\sigma), p_{N}\left(\sigma^{\prime}\right)\right\}=\delta_{N}^{M} \delta\left(\sigma-\sigma^{\prime}\right), \quad\left\{\omega(\sigma), \pi^{\omega}\left(\sigma^{\prime}\right)\right\}=\delta\left(\sigma-\sigma^{\prime}\right)
$$

we find

$$
\begin{aligned}
\left\{\mathbf{T}_{S}(\xi), \omega(\sigma)\right\} & =-\xi(\sigma) \partial_{\sigma} \omega(\xi)-2 \partial_{\sigma} \xi(\sigma) \omega(\sigma) \\
\left\{\mathbf{T}_{S}(\xi), \pi^{\omega}(\sigma)\right\} & =\partial_{\sigma} \xi(\sigma) \pi^{\omega}(\sigma)-\partial_{\sigma} \pi^{\omega}(\sigma) \xi(\sigma) \\
\left\{\mathbf{T}_{S}(\xi), p_{i}(\sigma)\right\} & =-\partial_{\sigma} \xi(\sigma) p_{i}(\sigma)-\xi(\sigma) \partial_{\sigma} p_{i}(\sigma) \\
\left\{\mathbf{T}_{S}(\xi), x^{i}(\sigma)\right\} & =-\partial_{\sigma} x^{i}(\sigma) \xi(\sigma) .
\end{aligned}
$$

Then we easily determine

$$
\left\{\mathbf{T}_{S}(\xi), \mathcal{H}_{\sigma}(\sigma)\right\}=-2 \partial_{\sigma} \xi(\sigma) \mathcal{H}_{\sigma}(\sigma)-\xi(\sigma) \partial_{\sigma} \mathcal{H}_{\sigma}(\sigma)
$$

and consequently

$$
\left\{\mathbf{T}_{S}(\xi), \mathbf{T}_{S}(\eta)\right\}=\int d \sigma\left(\xi \partial_{\sigma} \eta-\partial_{\sigma} \xi \eta\right) \mathcal{H}_{\sigma}(\sigma)=\mathbf{T}_{S}\left(\xi \partial_{\sigma} \eta-\partial_{\sigma} \xi \eta\right)
$$

As the next step we determine the Poisson bracket $\left\{\mathbf{T}_{S}(\xi), \mathbf{T}_{T}(n)\right\}$. Firstly, using (4.12) we find that

$$
\left\{\mathbf{T}_{S}(\xi), \mathcal{H}_{\tau}(\sigma)\right\}=-\partial_{\sigma} \xi(\sigma) \mathcal{H}_{\tau}(\sigma)-\xi(\sigma) \partial_{\sigma} \mathcal{H}_{\tau}(\sigma)
$$

so that

$$
\left\{\mathbf{T}_{S}(\xi), \mathbf{T}_{T}(n)\right\}=-\int d \sigma n(\sigma)\left(\partial_{\sigma} \xi \mathcal{H}_{\tau}+\xi \partial_{\sigma} \mathcal{H}_{\tau}\right)=\mathbf{T}_{T}\left(\xi \partial_{\sigma} n\right)
$$

As the final step we calculate the Poisson bracket $\left\{\mathbf{T}_{T}(f), \mathbf{T}_{T}(g)\right\}$ where we expect, with analogy with calculation of Poisson bracket of Hamiltonian constraints in Horava-Lifshitz gravity the problem with its closure. In fact, after some algebra we find following result 


$$
\begin{aligned}
& \left\{\mathbf{T}_{T}(f), \mathbf{T}_{T}(g)\right\}= \\
= & \int d \sigma\left(f \partial_{\sigma} g-\partial_{\sigma} f g\right)\left(\frac{2 \pi \alpha^{\prime}}{N^{2} \omega} F^{\prime}\left(p_{t}-N^{k} p_{k}\right) N^{i} h_{i j} \partial_{\sigma} x^{j}+\frac{1}{\omega} F^{\prime 2}(\ldots) p_{i} \partial_{\sigma} x^{i}\right) .
\end{aligned}
$$

This result shows that the algebra of constraints $\mathbf{T}_{T}$ is not closed. Then as in case of Hořava-Lifshitz gravity we see that the algebra closes when we impose the projectability condition on the lapse function $n_{\tau}$

$$
n_{\tau}=n(\tau) .
$$

Then it is easy to see that the algebra of constraints of LBS theory is closed and takes the form

$$
\begin{aligned}
\left\{\mathbf{T}_{S}(\xi), \mathbf{T}_{S}(\eta)\right\} & =\mathbf{T}_{S}\left(\xi \partial_{\sigma} \eta-\partial_{\sigma} \xi \eta\right) \\
\left\{\mathbf{T}_{S}(\xi), \mathbf{T}_{T}(f)\right\} & =0 \\
\left\{\mathbf{T}_{T}(g), \mathbf{T}_{T}(g)\right\} & =0
\end{aligned}
$$

where $\mathbf{T}_{T}(f)$ is defined

$$
\mathbf{T}_{T}=f(\tau) \int d \sigma \mathcal{H}_{\tau}(\sigma) .
$$

Let us now summarize consequences of the breaking of Lorentz invariance of the target space-time for the construction of the string action in given background. As the first one we have to demand that the spatial part of the world-sheet metric is dynamical. Secondly, the full world-sheet diffeomorphism is replaced with the world-sheet foliation preserving diffeomorphism. Finally, the requirement of the consistent Hamiltonian treatment of the LBS theory implies the necessity of the projectability condition on the lapse function.

\section{Acknowledgements:}

This work was supported by the Czech Ministry of Education under Contract No. MSM 0021622409.

\section{References}

[1] P. Horava, "Quantum Gravity at a Lifshitz Point," Phys. Rev. D 79 (2009) 084008 [arXiv:0901.3775 [hep-th]].

[2] P. Horava, "Membranes at Quantum Criticality," JHEP 0903 (2009) 020 [arXiv:0812.4287 [hep-th]].

[3] P. Horava, "Quantum Criticality and Yang-Mills Gauge Theory," arXiv:0811.2217 [hep-th].

[4] K. Koyama and F. Arroja, "Pathological behaviour of the scalar graviton in Horava-Lifshitz gravity," arXiv:0910.1998 [hep-th]. 
[5] M. i. Park, "Remarks on the Scalar Graviton Decoupling and Consistency of Horava Gravity," arXiv:0910.1917 [hep-th].

[6] B. Chen, S. Pi and J. Z. Tang, "Power spectra of scalar and tensor modes in modified Horava -Lifshitz gravity," arXiv:0910.0338 [hep-th].

[7] A. Wang, D. Wands and R. Maartens, "Scalar field perturbations in Horava-Lifshitz cosmology," arXiv:0909.5167 [hep-th].

[8] J. Z. Tang and B. Chen, "Static Spherically Symmetric Solutions to modified Horava-Lifshitz Gravity with Projectability Condition," arXiv:0909.4127 [hep-th].

[9] C. G. Boehmer and F. S. N. Lobo, "Stability of the Einstein static universe in IR modified Hořava gravity," arXiv:0909.3986 [gr-qc].

[10] G. Leon and E. N. Saridakis, "Phase-space analysis of Horava-Lifshitz cosmology," arXiv:0909.3571 [hep-th].

[11] D. Blas, O. Pujolas and S. Sibiryakov, "A healthy extension of Horava gravity," arXiv:0909.3525 [hep-th].

[12] P. Wu and H. W. Yu, "Stability of the Einstein static universe in Horava-Lifshitz gravity," arXiv:0909.2821 [gr-qc].

[13] L. Iorio and M. L. Ruggiero, "Horava-Lifshitz gravity and Solar System orbital motions," arXiv:0909.2562 [gr-qc].

[14] C. Ding, S. Chen and J. Jing, "Dynamical evolution of scalar perturbation in Horaava-Lifshitz black-hole spacetimes," arXiv:0909.2490 [gr-qc].

[15] S. Carloni, E. Elizalde and P. J. Silva, "An analysis of the phase space of Horava-Lifshitz cosmologies," arXiv:0909.2219 [hep-th].

[16] H. W. Lee, Y. W. Kim and Y. S. Myung, "Extremal black holes in the Horaava-Lifshitz gravity," arXiv:0907.3568 [hep-th].

[17] J. Kluson, "Horava-Lifshitz f(R) Gravity," arXiv:0907.3566 [hep-th].

[18] A. Wang and R. Maartens, "Linear perturbations of cosmological models in the Horava-Lifshitz theory of gravity without detailed balance," arXiv:0907.1748 [hep-th].

[19] J. J. Peng and S. Q. Wu, "Hawking Radiation of Black Holes in Infrared Modified Hoŕava-Lifshitz Gravity," arXiv:0906.5121 [hep-th].

[20] S. Mukohyama, "Caustic avoidance in Horava-Lifshitz gravity," JCAP 0909 (2009) 005 [arXiv:0906.5069 [hep-th]].

[21] D. Blas, O. Pujolas and S. Sibiryakov, "On the Extra Mode and Inconsistency of Horava Gravity," JHEP 0910 (2009) 029 [arXiv:0906.3046 [hep-th]].

[22] C. Germani, A. Kehagias and K. Sfetsos, "Relativistic Quantum Gravity at a Lifshitz Point," JHEP 0909 (2009) 060 [arXiv:0906.1201 [hep-th]].

[23] Y. S. Myung, "Propagations of massive graviton in the deformed Horava-Lifshitz gravity," arXiv:0906.0848 [hep-th].

[24] M. i. Park, "The Black Hole and Cosmological Solutions in IR modified Horava Gravity," JHEP 0909 (2009) 123 [arXiv:0905.4480 [hep-th]]. 
[25] M. Sakamoto, "Strong Coupling Quantum Einstein Gravity at a z=2 Lifshitz Point," Phys. Rev. D 79 (2009) 124038 [arXiv:0905.4326 [hep-th]].

[26] S. Nojiri and S. D. Odintsov, "Covariant Horava-like renormalizable gravity and its FRW cosmology," arXiv:0905.4213 [hep-th].

[27] G. Calcagni, "Detailed balance in Horava-Lifshitz gravity," arXiv:0905.3740 [hep-th].

[28] S. Mukohyama, "Dark matter as integration constant in Horava-Lifshitz gravity," Phys. Rev. D 80 (2009) 064005 [arXiv:0905.3563 [hep-th]].

[29] X. Gao, Y. Wang, R. Brandenberger and A. Riotto, "Cosmological Perturbations in Horava-Lifshitz Gravity," arXiv:0905.3821 [hep-th].

[30] Y. W. Kim, H. W. Lee and Y. S. Myung, "Nonpropagation of scalar in the deformed Hořava-Lifshitz gravity," arXiv:0905.3423 [hep-th].

[31] T. P. Sotiriou, M. Visser and S. Weinfurtner, "Quantum gravity without Lorentz invariance," JHEP 0910 (2009) 033 [arXiv:0905.2798 [hep-th]].

[32] M. Li and Y. Pang, "A Trouble with Hořava-Lifshitz Gravity," JHEP 0908 (2009) 015 [arXiv:0905.2751 [hep-th]].

[33] R. G. Cai, B. Hu and H. B. Zhang, "Dynamical Scalar Degree of Freedom in Horava-Lifshitz Gravity," Phys. Rev. D 80 (2009) 041501 [arXiv:0905.0255 [hep-th]].

[34] S. Mukohyama, K. Nakayama, F. Takahashi and S. Yokoyama, "Phenomenological Aspects of Horava-Lifshitz Cosmology," Phys. Lett. B 679 (2009) 6 [arXiv:0905.0055 [hep-th]].

[35] T. P. Sotiriou, M. Visser and S. Weinfurtner, "Phenomenologically viable Lorentz-violating quantum gravity," Phys. Rev. Lett. 102 (2009) 251601 [arXiv:0904.4464 [hep-th]].

[36] X. Gao, "Cosmological Perturbations and Non-Gaussianities in Hořava-Lifshitz Gravity," arXiv:0904.4187 [hep-th].

[37] Y. S. Piao, "Primordial Perturbation in Horava-Lifshitz Cosmology," arXiv:0904.4117 [hep-th].

[38] R. G. Cai, Y. Liu and Y. W. Sun, "On the $z=4$ Horava-Lifshitz Gravity," JHEP 0906 (2009) 010 [arXiv:0904.4104 [hep-th]].

[39] H. Nastase, "On IR solutions in Horava gravity theories," arXiv:0904.3604 [hep-th].

[40] R. Brandenberger, "Matter Bounce in Horava-Lifshitz Cosmology," Phys. Rev. D 80 (2009) 043516 [arXiv:0904.2835 [hep-th]].

[41] S. Mukohyama, "Scale-invariant cosmological perturbations from Horava-Lifshitz gravity without inflation," JCAP 0906 (2009) 001 [arXiv:0904.2190 [hep-th]].

[42] H. Lu, J. Mei and C. N. Pope, "Solutions to Horava Gravity," Phys. Rev. Lett. 103 (2009) 091301 [arXiv:0904.1595 [hep-th]].

[43] J. Kluson, "Branes at Quantum Criticality," JHEP 0907 (2009) 079 [arXiv:0904.1343 [hep-th]].

[44] E. Kiritsis and G. Kofinas, "Horava-Lifshitz Cosmology," Nucl. Phys. B 821 (2009) 467 [arXiv:0904.1334 [hep-th]].

[45] G. Calcagni, "Cosmology of the Lifshitz universe," JHEP 0909 (2009) 112 [arXiv:0904.0829 [hep-th]]. 
[46] T. Takahashi and J. Soda, "Chiral Primordial Gravitational Waves from a Lifshitz Point," Phys. Rev. Lett. 102 (2009) 231301 [arXiv:0904.0554 [hep-th]].

[47] M. Visser, "Lorentz symmetry breaking as a quantum field theory regulator," Phys. Rev. D 80 (2009) 025011 [arXiv:0902.0590 [hep-th]].

[48] A. A. Kocharyan, "Is nonrelativistic gravity possible?," Phys. Rev. D 80 (2009) 024026 [arXiv:0905.4204 [hep-th]].

[49] B. Chen, S. Pi and J. Z. Tang, "Scale Invariant Power Spectrum in Hořava-Lifshitz Cosmology without Matter," arXiv:0905.2300 [hep-th].

[50] E. N. Saridakis, "Horava-Lifshitz Dark Energy," arXiv:0905.3532 [hep-th].

[51] C. Bogdanos and E. N. Saridakis, "Perturbative instabilities in Horava gravity," arXiv:0907.1636 [hep-th].

[52] Y. F. Cai and E. N. Saridakis, "Non-singular cosmology in a model of non-relativistic gravity," arXiv:0906.1789 [hep-th].

[53] S. K. Chakrabarti, E. N. Saridakis and A. A. Sen, "A new approach to modified-gravity models," arXiv:0908.0293 [astro-ph.CO].

[54] T. Jacobson, "Extended Horava gravity and Einstein-aether theory," arXiv:1001.4823 [hep-th].

[55] M. Chaichian, S. Nojiri, S. D. Odintsov, M. Oksanen and A. Tureanu, "Modified $F(R)$ Horava-Lifshitz gravity: a way to accelerating FRW cosmology," arXiv:1001.4102 [hep-th].

[56] D. Blas, O. Pujolas and S. Sibiryakov, "Comment on 'Strong coupling in extended Horava-Lifshitz gravity'," arXiv:0912.0550 [hep-th].

[57] M. Henneaux, A. Kleinschmidt and G. L. Gomez, "A dynamical inconsistency of Horava gravity," arXiv:0912.0399 [hep-th].

[58] E. Kiritsis, "Spherically symmetric solutions in modified Horava-Lifshitz gravity," arXiv:0911.3164 [hep-th].

[59] A. Papazoglou and T. P. Sotiriou, "Strong coupling in extended Horava-Lifshitz gravity," arXiv:0911.1299 [hep-th].

[60] J. Kluson, "New Models of $f(R)$ Theories of Gravity," arXiv:0910.5852 [hep-th].

[61] A. Wang and Y. Wu, "Thermodynamics and classification of cosmological models in the Horava-Lifshitz theory of gravity," JCAP 0907 (2009) 012 [arXiv:0905.4117 [hep-th]].

[62] R. G. Cai and A. Wang, "Singularities in Horava-Lifshitz theory," arXiv:1001.0155 [hep-th].

[63] B. R. Majhi, "Hawking radiation and black hole spectroscopy in Horava-Lifshitz gravity," arXiv:0911.3239 [hep-th].

[64] J. Greenwald, A. Papazoglou and A. Wang, "Black holes and stars in Horava-Lifshitz theory with projectability condition," arXiv:0912.0011 [hep-th].

[65] A. Ghodsi and E. Hatefi, "Extremal rotating solutions in Horava Gravity," arXiv:0906.1237 [hep-th].

[66] A. Ghodsi, "Toroidal solutions in Horava Gravity," arXiv:0905.0836 [hep-th]. 
[67] R. A. Konoplya, "Towards constraining of the Horava-Lifshitz gravities," Phys. Lett. B 679, 499 (2009) [arXiv:0905.1523 [hep-th]].

[68] E. O. Colgain and H. Yavartanoo, "Dyonic solution of Horava-Lifshitz Gravity," JHEP 0908 (2009) 021 [arXiv:0904.4357 [hep-th]].

[69] R. G. Cai, L. M. Cao and N. Ohta, "Topological Black Holes in Horava-Lifshitz Gravity," Phys. Rev. D 80 (2009) 024003 [arXiv:0904.3670 [hep-th]].

[70] R. G. Cai, L. M. Cao and N. Ohta, "Thermodynamics of Black Holes in Horava-Lifshitz Gravity," Phys. Lett. B 679 (2009) 504 [arXiv:0905.0751 [hep-th]].

[71] R. G. Cai and N. Ohta, "Horizon Thermodynamics and Gravitational Field Equations in Horava-Lifshitz Gravity," arXiv:0910.2307 [hep-th].

[72] D. Capasso and A. P. Polychronakos, "Particle Kinematics in Horava-Lifshitz Gravity," arXiv:0909.5405 [hep-th].

[73] T. Suyama, "Notes on Matter in Horava-Lifshitz Gravity," arXiv:0909.4833 [hep-th].

[74] J. M. Romero, V. Cuesta, J. A. Garcia and J. D. Vergara, "Conformal Anisotropic Mechanics," arXiv:0909.3540 [hep-th].

[75] A. E. Mosaffa, "On Geodesic Motion in Horava-Lifshitz Gravity," arXiv:1001.0490 [hep-th].

[76] E. Kiritsis and G. Kofinas, "On Horava-Lifshitz 'Black Holes'," arXiv:0910.5487 [hep-th].

[77] S. K. Rama, "Particle Motion with Hor̆ava - Lifshitz type Dispersion Relations," arXiv:0910.0411 [hep-th]. 